\title{
Molecular characterization and copy number of SMN1, SMN2 and NAIP in Chinese patients with spinal muscular atrophy and unrelated healthy controls
}

\author{
Ping Fang ${ }^{1+}$, Liang $\mathrm{Li}^{1+}$, Jian Zeng ${ }^{2}$, Wan-Jun Zhou' ${ }^{1}$, Wei-Qing Wu ${ }^{3}$, Ze-Yan Zhong ${ }^{1}$, Ti-Zhen Yan ${ }^{4}$, Jian-Sheng Xie ${ }^{3}$, \\ Jing Huang ${ }^{5}$, Li Lin ${ }^{1}$, Ying Zhao ${ }^{6}$ and Xiang-Min Xü ${ }^{1^{*}}$
}

\begin{abstract}
Background: Spinal muscular atrophy (SMA) is caused by SMN1 dysfunction, and the copy number of SMN2 and NAIP can modify the phenotype of SMA. The aim of this study was to analyze the copy numbers and gene structures of SMA-related genes in Chinese SMA patients and unrelated healthy controls.

Methods: Forty-two Chinese SMA patients and two hundred and twelve unrelated healthy Chinese individuals were enrolled in our study. The copy numbers and gene structures of SMA-related genes were measured by MLPA assay.

Results: We identified a homozygous deletion of SMN1 in exons 7 and 8 in 37 of 42 patients (88.1\%); the other 5 SMA patients (11.9\%) had a single copy of SMN1 exon 8. The proportions of the 212 unrelated healthy controls with different copy numbers for the normal SMN1 gene were 1 copy in 4 individuals (1.9\%), 2 copies in 203 (95.7\%) and 3 copies in 5 (2.4\%). Three hybrid SMN genes and five genes that lack partial sequences were found in SMA patients and healthy controls. Distributions of copy numbers for normal SMN2 and NAIP were significantly different $(P<0.001)$ in people with and without SMA.
\end{abstract}

Conclusion: The copy numbers and gene structures of SMA-related genes were different in Chinese SMA patients and healthy controls.

Keywords: Chinese, MLPA, SMA, Gene copy number

\section{Background}

Spinal muscular atrophy (SMA) is one of the most common autosomal recessive diseases and is characterized by degeneration of spinal cord motor neurons, atrophy of skeletal muscles, and generalized weakness [1]. The incidence of SMA is approximately $1 / 6,000$ to $1 / 10,000$ live births, and the carrier frequency is about $1 / 42$ in the Chinese population [2]. SMA is divided into four clinical types according to age of onset and achieved motor

\footnotetext{
* Correspondence: gzxuxm@pub.guangzhou.gd.cn

${ }^{\dagger}$ Equal contributors

'Department of Medical Genetics, School of Basic Medical Sciences, Southern Medical University, Avenue North1838, Guangzhou, Guangdong, People's Republic of China

Full list of author information is available at the end of the article
}

function: (1) severe type I; (2) intermediate type II; (3) mild type III; and (4) adult-onset type IV.

SMA is caused by the dysfunction of the survival motor neuron $(S M N)$ gene on chromosome $5 \mathrm{q} 13.2$. The two versions of $S M N$, SMN1 and $S M N 2$, differ by only five nucleotides. SMN1 produces a full-length transcript that encodes functional SMN protein. About 94\% of SMA patients have a homozygous deletion of SMN1 exon 7. SMN1 can be absent because of deletion or SMN1-to-SMN2 conversion [3]. A single nucleotide transition in $S M N 2$ exon 7 (c.840C > T) relative to $S M N 1$ causes most of the $S M N 2$ pre-mRNA to lack exon 7 and encode nonfunctional SMN $\Delta 7$ protein [4]. However, about $10 \%$ of $S M N 2$ pre-mRNA is normal and can be translated into full-length SMN protein. SMN2 partially functionally compensates an SMN1 homozygous 
deletion [5]. Thus, the SMN2 copy number influences SMA severity. In addition to the $S M N$ genes, the neuronal apoptosis inhibitory protein (NAIP) gene, also located at chromosome 5q13.2, is an SMA disease-related gene. The copy number of NAIP is reported to be correlated with SMA severity. SMA patients with fewer copies of NAIP have more severe phenotypes than patients with more copies of NAIP [6,7]. Moreover, both SMN2 and NAIP copy numbers were associated with the onset age, risk of death and survival probability of SMA patients [8]. Therefore, elucidating the gene copy numbers and structures of SMN1, SMN2 and NAIP is important for analyzing the molecular mechanism of SMA and for SMA clinical diagnosis.

The SMN1, SMN2 and NAIP genes are all located at $5 \mathrm{q} 13.2$, an unstable chromosomal region that is prone to deletion, duplication, and gene conversion. Copy numbers of SMN1 and SMN2 vary in humans. In Chinese populations, up to 4 SMN1 or SMN2 genes are reported in both healthy people and SMA patients [2,9]. In addition, $S M N$ gene deletions and rearrangements are found in different populations $[6,7,10]$. Detecting copies and gene structures of SMA-related genes is difficult because of the high homology between SMN1 and SMN2. However, the multiple ligation-dependent probe amplification (MLPA) assay has been established for detecting deletions and duplications of SMA-related genes [11,12].

To determine the copy numbers and structures of SMA-related genes in a Chinese population, we analyzed SMN1, SMN2 and NAIP in 42 Chinese SMA patients and 212 Chinese healthy individuals using MLPA assays.

\section{Methods}

\section{Population and patient samples}

A total of 212 peripheral blood samples were obtained from unrelated healthy adults (108 men and 104 women) at the Third Affiliated Hospital of Southern Medical University. In addition, we enrolled 42 unrelated SMA patients from four hospitals (18 patients from the Fuzhou General Hospital of Nanjing Military Command, 18 from the Shenzhen Maternity and Child Healthcare Hospital, 4 from the Liuzhou Municipal Maternity and Child Healthcare Hospital, and 2 from the Prenatal Diagnosis Center of Dongguan Maternal and Child Health Hospital). All SMA patients were confirmed by MLPA assay to have a homozygous deletion of SMN1 gene exon 7. All healthy individuals and SMA patients were of Han ethnicity.

\section{Ethical approval}

Ethical approval for this study has been obtained from the Ethical Committee of the Southern Medical University as well as from partners including the Ethics Committee of the Third Affiliated Hospital of the Southern Medical University, the Ethics Committee of Fuzhou General Hospital of Nanjing Military Command, the Ethics Committee of Shenzhen Maternal and Child Health Care Hospital, the Ethics Committee of Liuzhou Maternal and Child Health Care Hospital and the Ethics Committee of Reproductive Medical Center of Dongguan Maternal and Child Health Care Hospital. Written informed consent was obtained from all participants or guardians prior to the study.

\section{MLPA technique}

Human DNA was extracted from leukocytes in peripheral blood using a standard phenol/chloroform method. SMN1, SMN2 and NAIP copy numbers were determined by an MLPA technique using SALSA P021-A2 SMA kits (MRC-Holland, Amsterdam, Netherlands) that amplify 37 regions throughout the genome for products between 140 and $463 \mathrm{bp}$. Of the amplified regions, 15 were target sequences at the SMA locus on 5q13.2 and 22 were reference sequences. Ten probes were complementary to SMN1, SMN2 and NAIP sequences. Because of the sequence similarity of SMN1 and SMN2, probes SMN1-1", SMN1-5", SMN1-6" and SMN1-8" were complementary to common regions in exons $1,4,6,8$ of either $S M N 1$ or SMN2. Probes SMN1-7 $7^{\#}$ and SMN1-8 ${ }^{\#}$ were specific for SMN1 in exons 7 and 8. Probes SMN2- $7^{\#}$ and SMN2- $8^{\#}$ were specific for $S M N 2$ in exons 7 and 8 . Probes NAIP-1 ${ }^{\#}$ and NAIP-08 ${ }^{\#}$ were specific for NAIP in exons 13 and 5 (Figure 1). MLPA assays are a multiplex PCR strategy with four steps: DNA denaturation and probe hybridization, ligation of two probes, PCR of ligated probes, and separation of amplified fragments and data analysis. All experimental procedures were carried out according to kit instructions. MLPA products were separated and quantified by capillary electrophoresis using an ABI 3130XL Genetic Analyzer with LIZ 500 as the internal size standard. Data were analyzed with the GeneMapper software v3.2 package (Applied Biosystems, Foster city, CA). Intrasample normalization was by dividing the peak area of each probe's amplification product by the total area of reference probes only. Intersample normalization was by dividing the intranormalized probe ratio for a sample by the average intranormalized probe ratio of two reference samples. Reference samples had two copies of SMN1, SMN2 and NAIP genes. Intervals for estimating the copy number of each probe were defined according to the predefined standards of Alías et al. [13].

\section{Statistical analysis}

The distributions of samples with different SMN2 and NAIP copy numbers from SMA patients and healthy individuals were compared using the Mann-Whitney $U$ test. A p-value of less than 0.05 was considered statistically significant. All statistical analyses used the SPSS software package (version 13.0, SPSS Inc., Chicago, IL). 


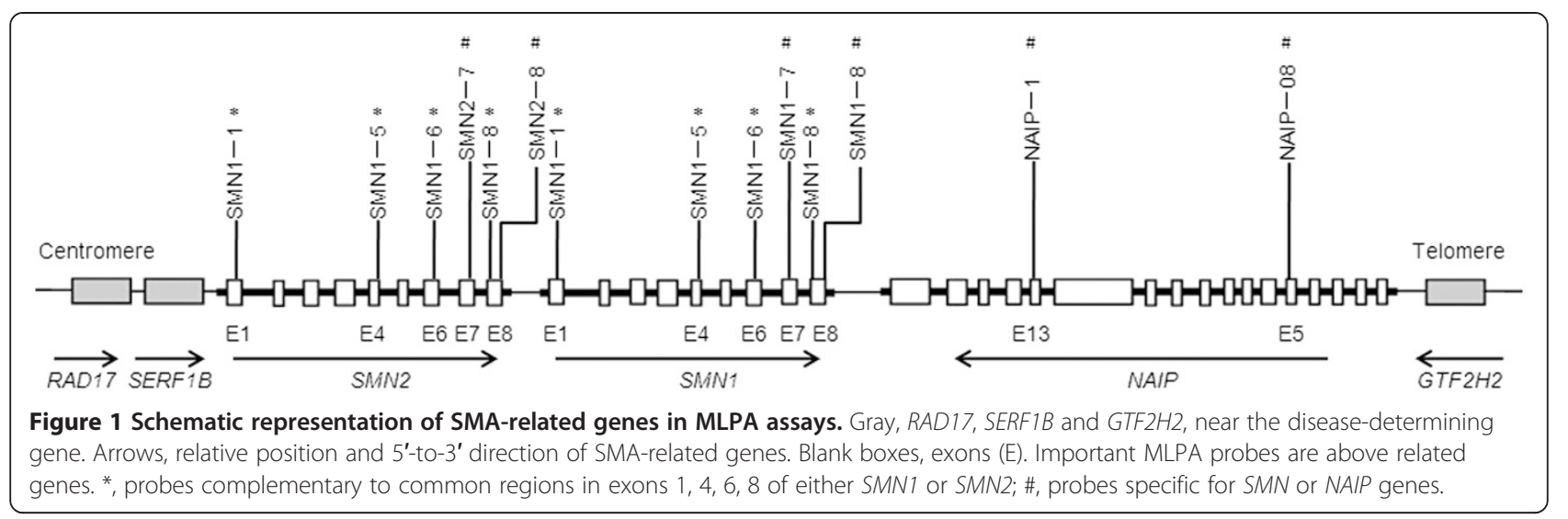

\section{Results}

\section{Gene copy number and structure in SMA patients}

All SMA patients had a homozygous deletion of SMN1 exon 7. We also identified a homozygous deletion of SMN1 exon 8 in 37 of 42 patients $(88.1 \%)$; the other 5 SMA patients $(11.9 \%)$ had a single copy of SMN1 exon 8. The proportions of SMA patients with various numbers of normal SMN2 copies were 1 copy in 2 patients (4.8\%), 2 copies in 14 (33.3\%), 3 copies in 24 (57.1\%) and 4 copies in $2(4.8 \%)$. The proportions of SMA patients with various numbers of normal NAIP were 0 copies in 4 patients (9.5\%), 1 copy in $26(61.9 \%)$ and 2 copies in 12 (28.6\%). In addition, 10 patients (23.8\%) had an NAIP gene lacking exon 5 (pattern $a$ in Figure 2), and 1 patient (2.4\%) had an $S M N 1$ gene lacking exon 7 (pattern $b$ in Figure 2). SMN2 genes lacking partial sequences were also found in SMA patients. Three patients (7.1\%) had an SMN2 lacking exon 1 to exon 7 (pattern $d$ in Figure 2), and two (4.8\%) had an SMN2 lacking exon 7 (pattern $c$ in Figure 2). We also found hybrid $S M N$ genes in SMA patients. A hybrid $S M N$ gene in which exon 8 of $S M N 2$ was converted to exon 8 of SMN1 (pattern $e$ in Figure 2) was found in 3 patients $(7.1 \%)$, and a hybrid $S M N$ gene in which exon 7 of SMN1 was converted to exon 7 of SMN2 (pattern $f$ in Figure 2) was found in 1 (2.4\%). MLPA probe copy numbers of 42 SMA patients are in Table 1 and inferred copy numbers are in Table 2. Abnormal gene structures are in Figure 2.

\section{Gene copy number and structure analysis of unrelated healthy individuals}

Using MLPA assays, 212 healthy Chinese participants were analyzed. We found that $117(55.2 \%)$ had normal gene copy numbers for SMN1/SMN2/NAIP (2/2/2) and 54 (25.5\%) had different gene copies numbers for SMN1/ SMN2 NAIP (2/1/2). The proportions of the 212 individuals with different copy numbers for the normal SMN1 gene were 1 copy in 4 individuals (1.9\%), 2 copies in 203 (95.7\%) and 3 copies in $5(2.4 \%)$. The proportions of the 212 with different gene copy numbers for normal SMN2 were 0 copies in 10 (4.7\%), 1 copy in 70 (33.0\%), 2 copies in $130(61.4 \%)$ and 3 copies in $2(0.9 \%)$. The proportions of the 212 with different gene copies for normal NAIP were 1 copy in 15 (7.1\%) and 2 copies in 197 (92.9\%). Genes lacking partial sequences were also found in healthy participants. Five $(2.4 \%)$ had an NAIP gene lacking exon 5

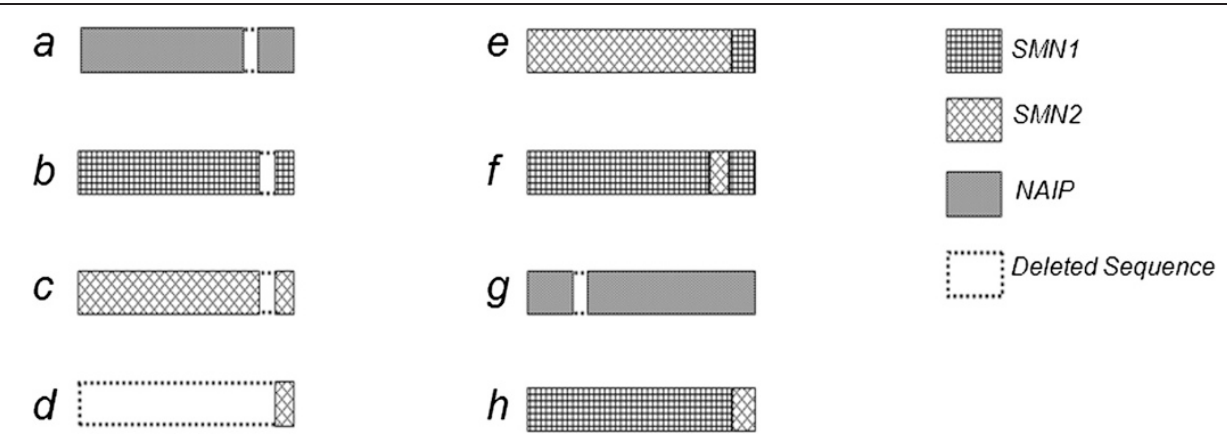

Figure 2 Gene structures inferred from MLPA results. (a) NAIP gene lacking exon 5; (b) SMN1 gene lacking exon 7; (c) SMN2 gene lacking exon 7; (d) SMN2 gene lacking exon 1 to exon 7; (e) Hybrid SMN gene in which exon 8 of SMN2 was converted to exon 8 of SMN1; (f) Hybrid SMN gene in which exon 7 of SMN1 was converted to exon 7 of SMN2; $(\boldsymbol{g})$ NAIP gene lacking exon13; (h) Hybrid SMN gene in which exon 8 of SMN1 was converted to exon 8 of SMN2. 
Table 1 The MLPA probe copy numbers of SMA-related genes in 42 Chinese SMA patients

\begin{tabular}{|c|c|c|c|c|c|c|c|c|c|c|c|}
\hline \multirow[b]{3}{*}{ Group } & \multirow{3}{*}{$\begin{array}{l}\text { Total } \\
\mathrm{N}=42\end{array}$} & \multicolumn{10}{|c|}{ MLPA probe copy number } \\
\hline & & \multicolumn{4}{|c|}{ SMN1/SMN2 } & \multicolumn{2}{|l|}{ SMN1 } & \multicolumn{2}{|l|}{ SMN2 } & \multicolumn{2}{|l|}{ NAIP } \\
\hline & & Exon1 & Exon4 & Exon6 & Exon8 & Exon7 & Exon8 & Exon7 & Exon8 & Exon 13 & Exon5 \\
\hline 1 & $3(7.1 \%)$ & 2 & 2 & 2 & 2 & 0 & 0 & 2 & 2 & 1 & 1 \\
\hline 2 & $3(7.1 \%)$ & 2 & 2 & 2 & 2 & 0 & 0 & 2 & 2 & 1 & 0 \\
\hline 3 & $1(2.4 \%)$ & 2 & 2 & 2 & 2 & 0 & 1 & 2 & 1 & 1 & 0 \\
\hline 4 & $1(2.4 \%)$ & 2 & 2 & 2 & 2 & 0 & 0 & 2 & 2 & 2 & 1 \\
\hline 5 & $1(2.4 \%)$ & 3 & 3 & $3-4$ & 3 & 0 & 0 & 3 & 3 & 2 & 1 \\
\hline 6 & $1(2.4 \%)$ & 3 & 3 & $3-4$ & 3 & 0 & 0 & 3 & 3 & 2 & 2 \\
\hline 7 & $4(9.5 \%)$ & 3 & 3 & 3 & $3-4$ & 0 & 0 & 3 & 3 & 2 & 2 \\
\hline 8 & $2(4.8 \%)$ & 4 & 4 & 4 & 4 & 0 & 0 & 4 & 4 & 2 & 2 \\
\hline 9 & $1(2.4 \%)$ & 3 & $3-4$ & 3 & 3 & 0 & 1 & 2 & 2 & 2 & 1 \\
\hline 10 & $2(4.8 \%)$ & 3 & $3-4$ & $3-4$ & 3 & 0 & 0 & 2 & 3 & 2 & 2 \\
\hline 11 & $9(21.3 \%)$ & 3 & $3-4$ & 3 & $3-4$ & 0 & 0 & 3 & 3 & 2 & 1 \\
\hline 12 & $6(14.2 \%)$ & 3 & 3 & 3 & 3 & 0 & 0 & 3 & 3 & 1 & 1 \\
\hline 13 & $1(2.4 \%)$ & 3 & 3 & 3 & 3 & 0 & 1 & 3 & 2 & 1 & 1 \\
\hline 14 & $1(2.4 \%)$ & 2 & 2 & 2 & 2 & 0 & 1 & 2 & 1 & 1 & 1 \\
\hline 15 & $1(2.4 \%)$ & $2-3$ & $2-3$ & 3 & 4 & 0 & 0 & 3 & 4 & 2 & 2 \\
\hline 16 & $2(4.8 \%)$ & $2-3$ & 3 & 3 & $3-4$ & 0 & 0 & 3 & 4 & 2 & 1 \\
\hline 17 & $2(4.8 \%)$ & $1-2$ & 2 & 2 & 2 & 0 & 0 & 2 & $2-3$ & 2 & 2 \\
\hline 18 & $1(2.4 \%)$ & $2-3$ & $2-3$ & 3 & 3 & 0 & 1 & 3 & 2 & 2 & 1 \\
\hline
\end{tabular}

Table 2 Copy numbers of SMA-related genes in 42 Chinese SMA patients determined by MLPA

\begin{tabular}{|c|c|c|c|c|c|c|c|}
\hline \multirow[b]{2}{*}{ Pattern } & \multirow[b]{2}{*}{ Group } & \multirow{2}{*}{$\begin{array}{l}\text { Total } \\
\mathrm{N}=42\end{array}$} & \multicolumn{5}{|l|}{ Gene copy number } \\
\hline & & & Normal SMN1 gene & Normal SMN2 gene & Normal NAIP gene & Hybrid SMN gene & Gene lacking partial sequences \\
\hline A & 1 & $3(7.1 \%)$ & 0 & 2 & 1 & 0 & 0 \\
\hline B & 2 & $3(7.1 \%)$ & 0 & 2 & 0 & 0 & $1^{a}$ \\
\hline C & 3 & $1(2.4 \%)$ & 0 & 1 & 0 & $1^{e}$ & $1^{a}$ \\
\hline D & 4 & $1(2.4 \%)$ & 0 & 2 & 1 & 0 & $1^{a}$ \\
\hline E & 5 & $1(2.4 \%)$ & 0 & 3 & 1 & 0 & $1^{a}$ \\
\hline $\mathrm{F}$ & $6 / 7$ & $5(11.9 \%)$ & 0 & 3 & 2 & 0 & 0 \\
\hline G & 8 & $2(4.8 \%)$ & 0 & 4 & 2 & 0 & 0 \\
\hline $\mathrm{H}$ & 9 & $1(2.4 \%)$ & 0 & 2 & 1 & 0 & $1^{a}$ and $1^{b}$ \\
\hline । & 10 & $2(4.8 \%)$ & 0 & 2 & 2 & 0 & $1^{c}$ \\
\hline J & 11 & $9(21.3 \%)$ & 0 & 3 & 1 & 0 & $1^{a}$ \\
\hline K & 12 & $6(14.2 \%)$ & 0 & 3 & 1 & 0 & 0 \\
\hline L & 13 & $1(2.4 \%)$ & 0 & 2 & 1 & $1^{e}$ & 0 \\
\hline M & 14 & $1(2.4 \%)$ & 0 & 1 & 1 & $1^{e}$ & 0 \\
\hline N & 15 & $1(2.4 \%)$ & 0 & 3 & 2 & 0 & $1^{d}$ \\
\hline $\mathrm{O}$ & 16 & $2(4.8 \%)$ & 0 & 3 & 1 & 0 & $1^{a}$ and $1^{d}$ \\
\hline P & 17 & $2(4.8 \%)$ & 0 & 2 & 2 & 0 & 0 \\
\hline Q & 18 & $1(2.4 \%)$ & 0 & 2 & 1 & $1^{f}$ & $1^{a}$ \\
\hline
\end{tabular}


(pattern $a$ in Figure 2) and 27 (12.7\%) had an NAIP gene lacking exon 13 (pattern $g$ in Figure 2). One person appeared to have an SMN1 gene lacking exon 7 (pattern $b$ in Figure 2) and an SMN2 gene lacking exon 7 (pattern $c$ in Figure 2). Three types of hybrid SMN genes were found in 6 of the healthy participants. Four (1.9\%) had a hybrid $S M N$ gene in which exon 8 of SMN2 was converted to exon 8 of SMN1 (pattern $e$ in Figure 2). Two (0.9\%) had a hybrid $S M N$ gene in which exon 8 of $S M N 1$ was converted to exon 8 of SMN2 (pattern $h$ in Figure 2). MLPA results for 212 healthy individuals are in Table 3 and inferred gene copy numbers are in Table 4. Abnormal gene structures are in Figure 2.

\section{Differences in distribution of participants by SMN2 and NAIP gene copy numbers}

We examined the distribution of different copy numbers for the normal SMN2 and NAIP genes in SMA patients and normal individuals. Distributions by copy number for normal SMN2 were significantly different in SMA patients compared to participants without SMA $(P<$ 0.001 ) (Table 5). Distributions by copy number for normal NAIP were significantly different for SMA patients compared to healthy participants $(P<0.001)$ (Table 6).

\section{Discussion}

In this study, the copy numbers for $S M N 1, S M N 2$ and NAIP were determined in Chinese SMA patients and healthy controls using MLPA assays and plausible gene structures were inferred. We found that 37 (88.1\%) people with SMA had deletions in both exon 7 and exon 8 of SMN1 and the other 5 SMA patients (11.9\%) had a single copy of SMN1 exon 8. These results were similar to several other studies in different populations $[14,15]$. Chen et al. reported that a distribution for SMN2 copy numbers in 94 Chinese SMA patients of 5 copies in 1 patient (1.1\%), 4 copies in 24 (25.5\%), 3 copies in 47 (50\%) and 2 copies in $22(23.4 \%)$. No patients with 0 or 1 copy of SMN2 were found in their population [16]. In addition, $\mathrm{Qu}$ et al. reported that a distribution for SMN2 copy numbers in 232 Chinese SMA patients of 4 SMN2 copies in 13 patients (5.6\%), 3 copies in 153(65.9\%), 2 copies in $66(28.5 \%)$ and no patients having only 0 or 1 copy of SMN2 [8]. In our results, the proportion of SMA patients with a single normal SMN2 copy was $4.8 \%$ and no SMA patients completely lacked copies of SMN2. Although two SMA patients had a single normal copy of SMN2, they also had a hybrid $S M N$ gene. These findings indicated that the proportion of SMA patients with 0 or 1 copy of SMN2

Table 3 The MLPA probe copy numbers of SMA-related genes in 212 Chinese healthy individuals

\begin{tabular}{|c|c|c|c|c|c|c|c|c|c|c|c|}
\hline \multirow[b]{3}{*}{ Group } & \multirow{3}{*}{$\begin{array}{l}\text { Total } \\
\mathrm{N}=212\end{array}$} & \multicolumn{10}{|c|}{ MLPA probe copy number } \\
\hline & & \multicolumn{4}{|c|}{ SMN1/SMN2 } & \multicolumn{2}{|l|}{ SMN1 } & \multicolumn{2}{|l|}{ SMN2 } & \multicolumn{2}{|l|}{ NAIP } \\
\hline & & Exon1 & Exon4 & Exon6 & Exon8 & Exon7 & Exon8 & Exon7 & Exon8 & Exon 13 & Exon5 \\
\hline 1 & $117(55.2 \%)$ & $4-5$ & $4-5$ & 4 & $4-5$ & 2 & 2 & 2 & 2 & 2 & 2 \\
\hline 2 & $1(0.5 \%)$ & 4 & 4 & 4 & 4 & 2 & 3 & 2 & 1 & 2 & 2 \\
\hline 3 & $1(0.5 \%)$ & 4 & $4-5$ & 4 & 4 & 2 & 1 & 2 & 3 & 2 & 2 \\
\hline 4 & $54(25.4 \%)$ & $3-4$ & $3-4$ & 3 & $3-4$ & 2 & 2 & 1 & 1 & 2 & 2 \\
\hline 5 & $1(0.5 \%)$ & 3 & 3 & 3 & 3 & 2 & 3 & $1-2$ & 0 & 2 & 2 \\
\hline 6 & $7(3.3 \%)$ & $3-4$ & 3 & $3-4$ & $3-4$ & 2 & 2 & 1 & 1 & 2 & 3 \\
\hline 7 & $1(0.5 \%)$ & $3-4$ & $3-4$ & $3-4$ & 3 & 2 & 2 & 1 & 1 & 2 & 4 \\
\hline 8 & $4(1.9 \%)$ & 2 & 2 & 2 & 2 & 2 & 2 & 0 & 0 & 1 & 2 \\
\hline 9 & $5(2.3 \%)$ & 2 & $2-3$ & $2-3$ & $2-3$ & 2 & 2 & 0 & 0 & 1 & 3 \\
\hline 10 & $1(0.5 \%)$ & 3 & 3 & $3-4$ & $3-4$ & 2 & 2 & 1 & 1 & 1 & 1 \\
\hline 11 & $5(2.3 \%)$ & 4 & $4-5$ & 4 & 4 & 2 & 2 & 2 & 2 & 2 & 3 \\
\hline 12 & $1(0.5 \%)$ & 4 & $4-5$ & 4 & $4-5$ & 2 & 1 & 2 & 3 & 2 & 3 \\
\hline 13 & $2(0.9 \%)$ & 4 & $4-5$ & 4 & $4-5$ & 2 & 3 & 2 & 1 & 2 & 3 \\
\hline 14 & $2(0.9 \%)$ & 4 & 4 & 4 & 4 & 3 & 3 & 1 & 1 & 2 & 2 \\
\hline 15 & $3(1.4 \%)$ & 4 & $4-5$ & 4 & $4-5$ & 2 & 2 & 2 & 2 & 2 & 1 \\
\hline 16 & $2(0.9 \%)$ & $4-5$ & 5 & 5 & $4-5$ & 2 & 2 & 3 & 3 & 2 & 2 \\
\hline 17 & $1(0.5 \%)$ & 4 & $4-5$ & 4 & 5 & 1 & 2 & 1 & 2 & 2 & 2 \\
\hline 18 & $1(0.5 \%)$ & $4-5$ & 5 & 5 & $4-5$ & 3 & 3 & 2 & 2 & 2 & 3 \\
\hline 19 & $1(0.5 \%)$ & $4-5$ & 5 & $4-5$ & 5 & 3 & 3 & 2 & 2 & 2 & 1 \\
\hline 20 & $1(0.5 \%)$ & 3 & $3-4$ & $3-4$ & $3-4$ & 1 & 1 & 2 & 2 & 2 & 1 \\
\hline 21 & $1(0.5 \%)$ & 4 & 4 & 4 & 4 & 3 & 3 & 1 & 1 & 2 & 3 \\
\hline
\end{tabular}


Table 4 Copy numbers of SMA-related genes in 212 Chinese healthy individuals determined by MLPA

\begin{tabular}{|c|c|c|c|c|c|c|c|}
\hline \multirow[b]{2}{*}{ Pattern } & \multirow[b]{2}{*}{ Group } & \multirow{2}{*}{$\begin{array}{l}\text { Total } \\
\mathrm{N}=212\end{array}$} & \multicolumn{5}{|c|}{ Gene copy number } \\
\hline & & & $\begin{array}{l}\text { Normal } \\
\text { SMN1 gene }\end{array}$ & $\begin{array}{l}\text { Normal } \\
\text { SMN2 gene }\end{array}$ & $\begin{array}{l}\text { Normal } \\
\text { NAIP gene }\end{array}$ & $\begin{array}{l}\text { Hybrid } \\
\text { SMN gene }\end{array}$ & $\begin{array}{l}\text { Gene lacking } \\
\text { partial sequences }\end{array}$ \\
\hline $\bar{A}$ & 1 & $117(55.2 \%)$ & 2 & 2 & 2 & 0 & 0 \\
\hline B & 2 & $1(0.5 \%)$ & 2 & 1 & 2 & $1^{e}$ & 0 \\
\hline C & 3 & $1(0.5 \%)$ & 1 & 2 & 2 & $1^{h}$ & 0 \\
\hline $\mathrm{D}$ & 4 & $54(25.5 \%)$ & 2 & 1 & 2 & 0 & 0 \\
\hline$E$ & 5 & $1(0.5 \%)$ & 2 & 0 & 2 & $1^{e}$ & 0 \\
\hline $\mathrm{F}$ & $6 / 7$ & $8(3.8 \%)$ & 2 & 1 & 2 & 0 & $1^{g}$ \\
\hline G & $8 / 9$ & $9(4.2 \%)$ & 2 & 0 & 1 & 0 & $1^{g}$ \\
\hline $\mathrm{H}$ & 10 & $1(0.5 \%)$ & 2 & 1 & 1 & 0 & 0 \\
\hline । & 11 & $5(2.3 \%)$ & 2 & 2 & 2 & 0 & $1^{g}$ \\
\hline J & 12 & $1(0.5 \%)$ & 1 & 2 & 2 & $1^{h}$ & $1^{g}$ \\
\hline K & 13 & $2(0.9 \%)$ & 2 & 1 & 2 & $1^{e}$ & $1^{g}$ \\
\hline$L$ & 14 & $2(0.9 \%)$ & 3 & 1 & 2 & 0 & 0 \\
\hline M & 15 & $3(1.4 \%)$ & 2 & 2 & 1 & 0 & $1^{a}$ \\
\hline $\mathrm{N}$ & 16 & $2(0.9 \%)$ & 2 & 3 & 2 & 0 & 0 \\
\hline $\mathrm{O}$ & 17 & $1(0.5 \%)$ & 1 & 1 & 2 & 0 & $1^{b}$ and $1^{c}$ \\
\hline P & 18 & $1(0.5 \%)$ & 3 & 2 & 2 & 0 & $1^{g}$ \\
\hline Q & 19 & $1(0.5 \%)$ & 3 & 2 & 1 & 0 & $1^{a}$ \\
\hline $\mathrm{R}$ & 20 & $1(0.5 \%)$ & 1 & 2 & 1 & 0 & $1^{a}$ \\
\hline$S$ & 21 & $1(0.5 \%)$ & 3 & 1 & 2 & 0 & $1^{g}$ \\
\hline
\end{tabular}

${ }^{a}$ :NAIP gene lacking exon $5,{ }^{b}:$ SMN1 gene lacking exon $7,{ }^{c}: S M N 2$ gene lacking exon $7,{ }^{e}:$ Hybrid SMN gene in which exon 8 of $S M N 2$ was converted to exon 8 of $S M N 1,{ }^{g}$ :NAIP gene lacking exon13, ${ }^{h}$ :Hybrid SMN gene in which exon 8 of SMN1 was converted to exon 8 of SMN2.

was low in Chinese SMA patients. Similar to the previous report, 2 patients $(4.8 \%)$ had 4 SMN2 copies, and no patients had 5 or 6 SMN2 copies. The results revealed that the proportion of SMA patients with more than 4 SMN2 copies was low in Chinese population. Most of Chinese SMA patients had 2 or 3 SMN2 copies. The distribution of normal copy numbers of NAIP in 42 SMA patients were: homozygous deletion (9.5\%), 1 copy (61.9\%), and 2 copies $(28.6 \%)$. This result is similar to data reported by another two studies of Chinese SMA patients [8,17]. Using conversions between SMN1 and SMN2 in exons 7 and 8, six possible hybrid $S M N$ genes were determined [10]. The hybrid SMN genes of patterns $e$ and $f$ have been reported in Chinese SMA patients $[10,16]$. In addition, four types of

Table 5 Comparison of the distribution of SMN2 copy number in SMA patients and healthy individuals

\begin{tabular}{llll}
\hline $\begin{array}{l}\text { Number of normal } \\
\text { SMN2 }\end{array}$ & $\begin{array}{l}\text { SMA patients } \\
\mathbf{( N = 4 2 )}\end{array}$ & $\begin{array}{l}\text { Healthy individuals } \\
\mathbf{( N = 2 1 2 )}\end{array}$ & $P$ \\
\hline 0 & $0(0 \%)$ & $10(4.7 \%)$ & \\
1 & $2(4.8 \%)$ & $70(33.1 \%)$ & $<0.001$ \\
2 & $14(33.3 \%)$ & $130(61.3 \%)$ & \\
3 & $24(57.1 \%)$ & $2(0.9 \%)$ & \\
4 & $2(4.8 \%)$ & $0(0 \%)$ & \\
\hline
\end{tabular}

genes lacking partial sequences were found in SMA patients in this study. Ten SMA patients had an NAIP gene lacking exon 5, and this type (pattern $a$ in Figure 2) of deletion was also found in several studies $[7,18,19]$. Three patients had an SMN2 lacking exon 1 to exon 7 (pattern $d$ in Figure 2), and two had an SMN2 lacking exon 7 (pattern $c$ in Figure 2). We also inferred that one patient had an SMN1 lacking exon 7 (pattern $b$ in Figure 2). For all we know, this study is the first report of three types of $S M N$ (pattern $b, c, d$ ) lacking partial sequence.

The SMA-related genes of 212 Chinese healthy individuals were analyzed using MLPA assays. Zhu et al. reported that $1 \%$ of healthy Chinese individuals have four SMN1 copies [2]. However, no individuals with 4 copies of $S M N 1$ were found in our study. In addition, we identified 4 participants with a single normal SMN1 copy,

Table 6 Comparison of the distribution of NAIP copy number in SMA patients and healthy individuals

\begin{tabular}{llll}
\hline $\begin{array}{l}\text { Number of } \\
\text { normal NAIP }\end{array}$ & $\begin{array}{l}\text { SMA patients } \\
(\mathbf{N}=\mathbf{4 2})\end{array}$ & $\begin{array}{l}\text { Healthy individuals } \\
\mathbf{( N = 2 1 2 )}\end{array}$ & $\boldsymbol{P}$ \\
\hline 0 & $4(9.5 \%)$ & $0(0 \%)$ & $<0.001$ \\
1 & $26(61.9 \%)$ & $15(7.1 \%)$ & \\
2 & $12(28.6 \%)$ & $197(92.9 \%)$ & \\
\hline
\end{tabular}


for a carrier frequency of $1.9 \%$ in our study. The reported frequency of SMA carriers is $2.4 \%$ in the general Chinese population $[2,16]$, therefore, our carrier frequency was lower. In contrast to the SMA patients, no homozygous deletions of NAIP were found in healthy Chinese participants. In addition to the hybrid $S M N$ gene $e$ in SMA patients, the hybrid SMN gene in which exon 8 of SMN1 was converted to exon 8 of SMN2 (pattern $h$ in Figure 2) was found in healthy participants; this type of the hybrid $S M N$ gene has been reported previously $[10,20]$. Four types of genes lacking partial sequences (pattern $a, b, c, g$ in Figure 2) were found in healthy individuals of our study. The proportion of healthy individuals $(2.4 \%)$ with an NAIP gene lacking exon 5 was lower than for SMA patients $(23.8 \%)$. This result might indicate that deletion of exon 5 in NAIP is involved in the molecular basis of SMA. Of the healthy participants, 27 (12.7\%) had an NAIP gene with an exon 13 deletion, and this type of NAIP gene was not found in SMA patients. One SMA carrier had a ratio of 1:2 for exon 7 and 8 of SMN1 and a ratio of 1:2 for exon 7 and 8 of SMN2. We hypothesized that she had an SMN1 lacking exon 7 (pattern $b$ in Figure 2) and an SMN2 lacking exon 7 (pattern $c$ in Figure 2).

The distributions of different copy numbers for normal SMN2 were compared between Chinese SMA patients and healthy individuals and distributions were significantly different $(P<0.001)$ between SMA patients and people without SMA (Table 5). Similarly, in a study of 108 SMA patients and 22 healthy controls, Crawford et al. found that the SMN2 copy number was significantly lower in control subjects [21]. Thus, SMA patients appeared to be prone to having more SMN2 copies than the controls. Because of the lack of SMN protein, fewer copies of SMN2 decrease the likelihood of survival to birth. This might explain the difference in the distribution of people with different SMN2 copy numbers. We also analyzed the distribution of copy numbers of normal NAIP in SMA patients and people without SMA and found significant differences $(P<0.001)$ (Table 6). Compared with healthy people, SMA patients had fewer NAIP copies. NAIP modifies the clinical phenotypes of SMA patients. Patients with fewer copies of NAIP might have more severe clinical symptoms of SMA [6,7]. This might be why that distribution of NAIP copies was significantly different between people with and without SMA. The NAIP gene locus is near the SMN1 gene locus, and both are located at 5q13.2. We determined that some SMA patients had a large deletion in the SMN1 and NAIP loci. This could partially explain why SMA patients had fewer NAIP copies than people without SMA.

Currently, SMN1 is recognized as an SMA causing gene. In contrast, the SMN2 and NAIP have been characterized as a modifying factor of the clinical severity of SMA. So, the clinical diagnosis and prenatal diagnosis of SMA are mainly based on the identification of SMN1 copy numbers and the situation of the SMN1 gene exon7 [22-24]. The copy numbers of SMN2 and NAIP were used to evaluate the clinical phenotype of SMA patients [25,26]. According to our results, the differences between the SMA patients and healthy controls were not only the distribution of SMA-related genes, the gene structures were also different. Moreover, the proportions of abnormal gene structures were high in both SMA patients (36\%) and healthy controls (17\%). Our data revealed that only confirming the gene copy numbers may not be sufficient to the clinical diagnosis, prenatal diagnosis, phenotype evaluation and carrier screening of SMA. The abnormal gene structures should be taken into account in the clinical diagnosis of SMA. So the analyses of SMA-related gene structures were also important to the molecular diagnostics of SMA patients and carriers.

At present, most of previous studies about Chinese SMA disease only focused on SMA patients or healthy population. There were few articles about comparison and analysis of SMA-related genes and gene structures in the Chinese people with and without SMA. In this study, we compared copy numbers of SMN1, SMN2 and NAIP between Chinese SMA patients and healthy controls and inferred gene structures. We summarized the subdivision of SMA-related gene patterns (Tables 2 and 4) based on the MLPA technique. The genetic characteristics would be helpful for the further identification of the clinical subdivision of SMA patients. There are limitations of our study. All gene copy numbers and gene structures were inferred from MLPA results, so we didn't know the detailed haplotype of each participant. Therefore, further investigations are needed.

\section{Conclusions}

In conclusion, we analyzed and compared gene copy numbers and gene structures in Chinese SMA patients and healthy individuals. For the first time three types of $S M N$ lacking partial sequence were found. The distributions of different copy numbers for normal SMN2 and NAIP were significantly different between SMA patients and healthy controls. The gene structures of SMN and NAIP were also different between the SMA patients and healthy controls. Further studies are required to address the molecular mechanisms and clinical diagnoses of SMA.

\section{Abbreviations}

MLPA: Multiple ligation-dependent probe amplification; NAIP: Neuronal apoptosis inhibitory protein; SMA: Spinal muscular atrophy; SMN: Survival motor neuron.

\section{Competing interests}

The authors declare that they have no competing interests. 


\section{Authors' contributions}

XMX conceived and coordinated the study. L Li designed the experiments, analyzed the data and prepared the manuscript. PF, ZYZ and L Lin performed the experiments, assisted with data analysis and manuscript preparation. WJZ and JH recruited the healthy participants. JZ, WQW, TZY, JSX and YZ recruited the SMA patients and collected the samples. All authors read and approved the final manuscript.

\section{Acknowledgements}

This work was partially supported by the National Natural Science Fund of China (NSFC, No. 81101328), Pearl River S\&T Nova Program of Guangzhou (No. 2013 J2200050), the Natural Science Fund of Guangdong (No. S2012040007745) and the research fund for the distinguished young teachers of Southern Medical University to Liang Li.

\section{Author details}

'Department of Medical Genetics, School of Basic Medical Sciences, Southern Medical University, Avenue North1838, Guangzhou, Guangdong, People's Republic of China. 'Department of Clinical Laboratory, The Fuzhou General Hospital, Nanjing Military Command, Fuzhou, Fujian, People's Republic of China. ${ }^{3}$ Prenatal Diagnosis Center, Shenzhen Maternity and Child Healthcare Hospital, Shenzhen, Guangdong, People's Republic of China. ${ }^{4}$ Liuzhou Key Laboratory of birth defects prevention and control, Liuzhou Municipal Maternity and Child Healthcare Hospital, Liuzhou, Guangxi, People's Republic of China. ${ }^{5}$ The Third Affiliated Hospital of Southern Medical University, Guangzhou, Guangdong, People's Republic of China. ${ }^{6}$ Prenatal Diagnostic Center, Dongguan Maternal and Children Health Hospital, Dongguan, Guangdong, People's Republic of China.

Received: 22 August 2014 Accepted: 12 January 2015

Published online: 07 February 2015

\section{References}

1. Lunn MR, Wang CH. Spinal muscular atrophy. Lancet. 2008:371:2120-33.

2. Sheng-Yuan Z, Xiong F, Chen YJ, Yan TZ, Zeng J, Li L, et al. Molecular characterization of SMN copy number derived from carrier screening and from core families with SMA in a Chinese population. Eur J Hum Genet. 2010;18:978-84.

3. Ogino S, Wilson RB. Spinal muscular atrophy: molecular genetics and diagnostics. Expert Rev Mol Diagn. 2004;4:15-29.

4. Vitte J, Fassier C, Tiziano FD, Dalard C, Soave S, Roblot N, et al. Refined characterization of the expression and stability of the SMN gene products. Am J Pathol. 2007;171:1269-80.

5. Elsheikh B, Prior T, Zhang X, Miller R, Kolb SJ, Moore D, et al. An analysis of disease severity based on SMN2 copy number in adults with spinal muscular atrophy. Muscle Nerve. 2009:40:652-6.

6. Watihayati MS, Zabidi-Hussin AM, Tang TH, Matsuo M, Nishio H, Zilfalil BA. Deletion analyses of SMN1 and NAIP genes in Malaysian spinal muscular atrophy patients. Pediatr Int. 2007:49:4-11.

7. Omrani $\mathrm{O}$, Bonyadi M, Barzgar M. Molecular analysis of the SMN and NAIP genes in Iranian spinal muscular atrophy patients. Pediatr Int. 2009;51:193-6.

8. Qu YJ, Ge XS, Bai JL, Wang LW, Cao YY, Lu YY, et al. Association of Copy Numbers of Survival Motor Neuron Gene 2 and Neuronal Apoptosis Inhibitory Protein Gene With the Natural History in a Chinese Spinal Muscular Atrophy Cohort. J Child Neurol. 2014; Epub ahead of print.

9. Gong B, Zhang L, Hou YP, Hu HY, Li HC, Tan MY, et al. Carrier screening for spinal muscular atrophy in 4719 pregnant women in Shanghai region. Zhonghua Yi Xue Yi Chuan Xue Za Zhi. 2013;30:670-2.

10. Wang CC, Jong YJ, Chang JG, Chen YL, Wu SM. Universal fluorescent multiplex PCR and capillary electrophoresis for evaluation of gene conversion between SMN1 and SMN2 in spinal muscular atrophy. Anal Bioanal Chem. 2010;397:2375-83.

11. Arkblad EL, Darin N, Berg K, Kimber E, Brandberg G, Lindberg C, et al. Multiplex ligation-dependent probe amplification improves diagnostics in spinal muscular atrophy. Neuromuscul Disord. 2006;16:830-8.

12. Scarciolla O, Stuppia L, De Angelis MV, Murru S, Palka C, Giuliani R, et al. Spinal muscular atrophy genotyping by gene dosage using multiple ligation-dependent probe amplification. Neurogenetics. 2006:7:269-76.

13. Alías L, Bernal S, Barceló MJ, Also-Rallo E, Martínez-Hernández R, RodríguezAlvarez FJ, et al. Accuracy of marker analysis, quantitative real-time polymerase chain reaction, and multiple ligation-dependent probe amplification to determine SMN2 copy number in patients with spinal muscular atrophy. Genet Test Mol Biomarkers. 2011:15:587-94.

14. Liang $Y H$, Chen XL, Yu ZS, Chen CY, Bi S, Mao LG, et al. Deletion analysis of SMN1 and NAIP genes in Southern Chinese children with spinal muscular atrophy. J Zhejiang Univ Sci B. 2009;10:29-34.

15. Jedrzejowska M, Milewski M, Zimowski J, Borkowska J, Kostera-Pruszczyk A, Sielska D, et al. Phenotype modifiers of spinal muscular atrophy: the number of SMN2 gene copies, deletion in the NAIP gene and probably gender influence the course of the disease. Acta Biochim Pol. 2009;56:103-8.

16. Chen TH, Tzeng CC, Wang CC, Wu SM, Chang JG, Yang SN, et al. Identification of bidirectional gene conversion between SMN1 and SMN2 by simultaneous analysis of SMN dosage and hybrid genes in a Chinese population. J Neurol Sci. 2011;308:83-7.

17. He J, Zhang QJ, Lin QF, Chen YF, Lin XZ, Lin MT, et al. Molecular analysis of SMN1, SMN2, NAIP, GTF2H2, and H4F5 genes in 157 Chinese patients with spinal muscular atrophy. Gene. 2013;518:325-9.

18. Chang JG, Jong YJ, Huang JM, Wang WS, Yang TY, Chang CP, et al. Molecular basis of spinal muscular atrophy in Chinese. Am J Hum Genet 1995:57:1503-5.

19. Derakhshandeh-Peykar P, Esmaili M, Ousati-Ashtiani Z, Rahmani M, Babrzadeh F, Farshidi S, et al. Molecular analysis of the SMN1 and NAIP genes in Iranian patients with spinal muscular atrophy. Ann Acad Med Singapore. 2007;36:937-41

20. Burghes $\mathrm{AH}$. When is a deletion not a deletion? When it is converted. Am J Hum Genet. 1997;61:9-15.

21. Crawford TO, Paushkin SV, Kobayashi DT, Forrest SJ, Joyce CL, Finkel RS, et al. Pilot Study of Biomarkers for Spinal Muscular Atrophy Trial Group. PLoS One. 2012;7(4):e33572.

22. Prior TW. Spinal muscular atrophy diagnostics. J Child Neurol. 2007;22:952-6.

23. Maranda B, Fan L, Soucy JF, Simard L, Mitchell GA. Spinal muscular atrophy: clinical validation of a single-tube multiplex real time PCR assay fordetermination of SMN1 and SMN2 copy numbers. Clin Biochem. 2012;45:88-91.

24. Khaniani MS, Derakhshan SM, Abasalizadeh S. Prenatal diagnosis of spinal muscular atrophy: clinical experience and molecular genetics of SMN gene analysis in 36 cases. J Prenat Med. 2013;7:32-4

25. Harada Y, Sutomo R, Sadewa AH, Akutsu T, Takeshima Y, Wada H, et al. Correlation between SMN2 copy number and clinical phenotype of spinal muscular atrophy: three SMN2 copies fail to rescue some patients from the disease severity. J Neurol. 2002;249:1211-9.

26. Watihayati MS, Fatemeh $H$, Marini M, Atif AB, Zahiruddin WM, Sasongko $T H$, et al. Combination of SMN2 copy number and NAIP deletion predicts disease severity in spinal muscular atrophy. Brain Dev. 2009;31:42-5.

\section{Submit your next manuscript to BioMed Central and take full advantage of:}

- Convenient online submission

- Thorough peer review

- No space constraints or color figure charges

- Immediate publication on acceptance

- Inclusion in PubMed, CAS, Scopus and Google Scholar

- Research which is freely available for redistribution 